\title{
Can a Massive Graviton be a Stable Particle
}

\author{
Andrew Beckwith \\ Department of Physic, Chongquing University, Chongqing, China \\ E-mail: abeckwith@uh.edu \\ Received January 26, 2011; revised April 1, 2011; accepted April 3, 2011
}

\begin{abstract}
This document is based on a question asked in the Dark Side of the Universe 2010 conference in Leon, Mexico, when a researcher from India asked the author about how to obtain a stability analysis of massive gravitons. The answer to this question involves an extension of the usual Pauli_Fiertz Langrangian as written by Ortin, with non-zero graviton mass contributing to a relationship between the trace of a revised GR stress-energy tensor (assuming non-zero graviton mass), and the trace of a revised symmetric tensor times a tiny mass for a 4 dimensional graviton. The resulting analysis makes use of Visser's treatment of a stress energy tensor, with experimental applications discussed in the resulting analysis. If the square of frequency of a massive graviton is real valued and greater than zero, stability can be possibly confirmed experimentally.
\end{abstract}

Keywords: Graviton Stability, Gr Stress-Energy Tensor, 4 Dimensional Graviton

\section{Introduction}

The supposition advanced in this article is that initial relic energy flux is central to making predictions as to $S_{\text {entropy }} \sim n_{f}$, where $n_{f}$ is a "particle count" per phase space "volume" in the beginning of inflation [1,2]. So is $n_{f}$ due to gravitons in near-relic conditions? If so, can the gravitons carry information? Where $\left.S_{\text {entropy }} \sim n_{f}\right|_{\text {start-of-inf }}$ is about $10^{7}$ the value of entropy information, which is $\propto 10^{10}$ bits of "information" in line with Smoot's [3] talk at the Paris observatory. Having said that, a relevant issue raised in DSU 2010 is: if gravitons with a small mass are part of the bridge between

$$
\left.S_{\text {entropy }} \sim n_{f}\right|_{\text {start-of-inf }} \propto 10^{7} \Leftrightarrow \text { initial } \propto 10^{10} \text { informa- }
$$

tion bits, can one make a statement about necessary conditions for "massive" graviton stability? The conclusion is that stability of a massive graviton needs the square of frequency to be positive real valued.

\section{What can be Said about Massive Graviton Stability? Necessary Conditions}

This document looks at work presented by Maggiore [4], which specifically delineated for non-zero graviton mass, where $h \equiv \eta^{u v} h_{u v}=\operatorname{Trace} \cdot\left(h_{u v}\right)$ and $v$, that a small rest mass of the graviton is proportional to the trace of the energy-momentum tensor of general relativity.

$$
-3 m_{\text {graviton }}^{2} h=\frac{\kappa}{2} \times T
$$

This document uses Visser's [5] analysis of non-zero graviton mass for both $T$ and $h$. Equation (1) is used with particle count $n_{f}$ as a way to present initial GW relic inflation density using the definition given by Maggiore [4] as a way to state that a particle count:

$$
\begin{aligned}
\Omega_{g w} & \equiv \frac{\rho_{g w}}{\rho_{c}} \equiv \int_{f=0}^{f=\infty} \mathrm{d}(\log f) \times \Omega_{g w}(f) \Rightarrow h_{0}^{2} \Omega_{g w}(f) \\
& \cong 3.6 \times\left[\frac{n_{f}}{10^{37}}\right] \times\left(\frac{f}{1 \mathrm{kHz}}\right)^{4}
\end{aligned}
$$

where $n_{f}$ is the frequency-based numerical count of gravitons per unit phase space. To do so, let us give the reasons for using Visser's [5] values for $T$ and $h$, in Equation (1).

While Maggiore's [4] explanation, and his treatment of gravitational wave density is very good, the problem we have is that any relic conditions for GW involve stochastic background, and also that many theorists have relied upon turbulence and or other forms of plasmainduced generation of shock waves, as stated by Duerrer and Rinaldi [6] and others, looking at the electroweak transition as a $\mathrm{GW}$ generator. If relic conditions can also yield GW/graviton production, and the consequences 
exist up to the present era, then the question of stability of gravitons is even more essential. The author [1,2] uses a modification of results from Alcubierre [7], as an energy flux value for GW/gravitons, given by the Equation (3).

$$
E_{\text {initial-flux }} \cong\left[\frac{r^{2}}{64 \pi}\right] \times\left|+\partial_{r}^{2} h^{+}\right|^{2} \cdot\left[\tilde{n} \cdot t_{\text {Planck }}\right]^{3} \times \Omega_{\text {effective }}
$$

The $n_{f}$ value obtained, was used to make a relationship, using $\mathrm{Ng}$ 's [8] entropy counting algorithm of roughly $S_{\text {entropy }} \sim n_{f}$. The author suggests that in order to obtain $S_{\text {entropy }} \sim n_{f}$ from initial graviton production, as a way to quantify $n_{f}$, that a small mass of the graviton can be assumed. A small mass graviton in four dimensions only makes sense if it is a stable construct. The remainder of this article will give specific cases to provide criteria for stability for the low mass 4 dimensional graviton in obtaining his value of $S_{\text {entropy }} \sim n_{f}$ tying in what Ng's [8] entropy values as given by formulations started by $\mathrm{Ng}$ [8] and resultant information content present in the early universe. In doing so, the author will address if the correspondence principle and the closeness of the links to massless formalism of the graviton is due to 't Hoofts $[9,10]$; an idea of embedding QM within deterministic quantum theory, involving an embedding of quantum physics within a slightly "larger" highly non linear structure $[1,2]$.

\subsection{Defining the Graviton Problem and Using Visser's (1998) Inputs into $T_{u v}$}

This section defines a graviton in terms of a dark matter component, and an effective dark energy contribution. To do this, we look at a modification of what was presented by Maartens [11], which is written as Equation (4) below. Here we are actually using

$$
m_{n}(\text { Graviton })=\frac{n}{L}+10^{-65} \text { grams }
$$

Equation (4) is a simplification of what is written for a Kaluza Klein particle, which in this case would read as given by Sarkar [1,2]

$$
m_{n}(\text { graviton })=\sqrt{\left(\frac{n}{L}\right)^{2}+\left(10^{-65} \text { grams }\right)^{2}}
$$

On the face of it, this assignment of a mass of about $10^{-65}$ grams for a 4 dimensional graviton, allowing for $m_{0}($ Graviton- $4 D) \sim 10^{-65}$ grams violates all known quantum mechanics, and is to be avoided. Numerous authors, including Maggiore [4], have richly demonstrated how adding a term to the Fiertz Lagrangian as written up by Ortin [13] (2007) for gravitons, and assuming massive gravitons, leads to results which appear to violate field theory. Turning to the problem, we can examine what inputs to Equation (1) can tell us about whether there are grounds for Equation (4) and what this says about measurement protocol for both GW and gravitons as given in Equation (2). Visser (1998) came up with inputs into the GR stress tensor and also for the perturbing term $h_{u v}$ which will be given below. We will use them in conjunction with Equation (1) to perform a stability analysis of the consequences of setting the value of $m_{0}($ Graviton- $4 D) \sim 10^{-65}$ grams and from there discuss how to used 't Hooft's $(2002,2006)$ supposition of deterministic QM, as an embedding of QFT, and more could play a role if there are conditions for stability of $m_{0}($ Graviton- $4 D) \sim 10^{-65}$ grams.

\subsection{Visser's Treatment of the Stress Energy Tensor of GR, and Its Applications}

This section will derive stability conditions for the graviton, if the graviton has a small rest mass. Visser [5] stated a stress energy tensor treatment of gravitons along the lines of a four dimensional matrix treatment of the stress energy tensor as given by

$$
\begin{aligned}
& \left.m_{0}(\text { Graviton- } 4 D) \sim 10^{-65} T_{u v}\right|_{m \neq 0} \\
& =\left[\left(\frac{\hbar}{l_{P}^{2} \lambda_{g}^{2}}\right) \times\left(\frac{G M}{r}\right) \times \exp \left(\frac{r}{\lambda_{g}}\right)+\left(\frac{G M}{r}\right)^{2}\right] \\
& \times\left[\begin{array}{llll}
4 & 0 & 0 & 0 \\
0 & 0 & 0 & 0 \\
0 & 0 & 0 & 0 \\
0 & 0 & 0 & 0
\end{array}\right]
\end{aligned}
$$

Furthermore, his version of $g_{u v}=\eta_{u v}+h_{u v}$ can be written as setting

$$
h_{u v} \equiv 2 \frac{G M}{r} \mp\left[\exp \left(\frac{-m_{g} r}{\hbar}\right)\right] \times\left(2 \times V_{\mu} V_{v}+\eta_{u v}\right)
$$

If one adds in velocity "reduction" put in for speed of gravitons as stated by Visser [5]

$$
v_{g}=c \times \sqrt{1-\frac{m_{g}^{2} \times c^{4}}{\hbar^{2} \omega_{g}^{2}}}
$$

As well as often setting $(M G / r) \approx 1 / 5$ for reasons which Visser [5] outlined, one can insert all this into Equation (1) to obtain a real value for the square of frequency $>0$, i.e.

$$
\begin{aligned}
& \hbar^{2} \omega^{2} \cong m_{g}^{2} c^{4} \times[1 /(1-\breve{A})]>0 ; \\
\breve{A} & =\left\{1-\frac{1}{6 m_{g} c^{2}}\left(\frac{\hbar^{2}}{l_{P}^{2} \lambda_{g}^{2}} \times \exp \left[-\frac{r}{\lambda_{g}}+\frac{m_{g} \times r}{\hbar}\right]\right.\right. \\
& \left.\left.+\left(\frac{M G}{r}\right) \times \exp \left(\frac{m_{g} r}{\hbar}\right)\right)\right\}^{2}
\end{aligned}
$$


According to $\Omega_{g w} \equiv \rho_{g w} / \rho_{c}$ by Kim [14], if the square of frequency of a graviton, with mass, is $>0$, and real valued, it is likely that the graviton is stable, at least with regards to perturbations. Kim's [14] article is with regards to Gravitons in brane/string theory, but it is likely that the same dynamic for semi classical representations of a graviton with mass.

Conditions permitting equation (8) to have positive values - This section is to obtain sufficient conditions for stability of a graviton. Looking at Equation (8) is the same as analyzing how

$$
\begin{aligned}
\breve{A}= & \left\{1-\frac{1}{6 m_{g} c^{2}}\left(\frac{\hbar^{2}}{l_{P}^{2} \lambda_{g}^{2}} \times \exp \left[-\frac{r}{\lambda_{g}}+\frac{m_{g} \times r}{\hbar}\right]\right.\right. \\
& \left.+\left(\frac{M G}{r}\right) \times \exp \left(\frac{m_{g} r}{\hbar}\right)\right\}^{2}<1
\end{aligned}
$$

That is, setting

$$
\begin{aligned}
0< & \frac{1}{6 m_{g} c^{2}}\left(\frac{\hbar^{2}}{l_{P}^{2} \lambda_{g}^{2}} \times \exp \left[-\frac{r}{\lambda_{g}}+\frac{m_{g} \times r}{\hbar}\right]\right. \\
& \left.+\left(\frac{M G}{r}\right) \times \exp \left(\frac{m_{g} r}{\hbar}\right)\right)<1
\end{aligned}
$$

Note that Visser [5] writes

$m_{g}<2 \times 10^{-29} \mathrm{eV} \sim 2 \times 10^{-38} m_{\text {nucleon }}$, and a wavelength $\lambda_{g}^{g} \sim 6 \times 10^{22}$ meters. The two values, as well as ascertaining when one can use $M G / r \sim 1 / 5$, with $r$ the usual distance from a graviton generating source, and $\mathrm{M}$ the mass' of an object which would be a graviton emitter put severe restrictions as to the volume of space time values for which $r$ could be ascertained. If, however, Equation (10) had, in most cases, a setting for which, then in many cases, Equation (8) would hold

$$
0 \leq \exp \left[-\frac{r}{\lambda_{g}}+\frac{m_{g} \times r}{\hbar}\right] \ll 1 .
$$

The author believes that such a configuration would be naturally occurring in most generation of gravitons at, or before the electro weak transition point in early cosmology evolution. The author believes, that Equation (12) would allow to predict a particle count behavior along the lines of $n_{f} \approx 10^{6}$ to $10^{7}$, which is put into Equation (2) and has implications for what to look for in stochastic GW generation.

\subsection{Revisiting Ng's Counting Algorithm for Entropy, and Graviton Mass}

The wavelength for a graviton as may be chosen to do such an information exchange would be part of a graviton as being part of an information counting algorithm. Namely argue that when taking the log, that the $1 / N$ term drops out. As used by $\mathrm{Ng}$ [8]

$$
Z_{N} \sim(1 / N !) \times\left(V / \lambda^{3}\right)^{N}
$$

This, according to $\mathrm{Ng}$ [8] leads to entropy of the limiting value of, if $S=\left(\log \left[Z_{N}\right]\right)$ will be modified by having the following done, namely after his use of quantum infinite statistics,

$$
S \approx N \times\left(\log \left[V / \lambda^{3}\right]+5 / 2\right) \approx N
$$

Eventually, the author hopes to put on a sound foundation what ' $t$ Hooft $[9,10]$ is doing with respect to deterministic quantum mechanics and equivalence classes embedding quantum particle structures. Furthermore, making a count of gravitons with $S \approx N \sim 10^{7}$ gravitons', with Lloyd's [15] formalism

$$
I=S_{\text {total }} / k_{B} \ln 2=[\text { \#operations }]^{3 / 4} \sim 10^{7}
$$

as implying at least one operation per unit graviton, with gravitons being one unit of information, per produced graviton. Note, Smoot [3] gave initial values of the operations as

$$
[\text { \# operations }]_{\text {initially }} \sim 10^{10}
$$

The author's work tends to support this value, and if gravitons are indeed stable in initial conditions, information exchange between a prior to a present universe may become a topic of experimental investtigation.

\section{Conclusion}

The author pursued this question, partly due to wishing to determine if a non brane theory way to identify graviton stability existed. The author was particularly impressed with Visser's [5] treatment of gravitons in the context of both an alleged graviton wave length, and the net slow down of gravitons, as referenced in Equation (7). Note, that the treatment of Equation (6) above heavily depends upon a small mass to the graviton very slightly lowering the speed of graviton to just below the speed of light. As the graviton mass is slight, the velocity of a spin two graviton is ALMOST the speed of light. If Equation (12) is verified in measurement, and there is a search done for regions of space time for graviton production, then the author hopes for a refinement and vetting experimentally as to Duerrer's and Rinaldi [6] supposition of turbulence in the electro weak transition being the major source for $\mathrm{GW} /$ graviton production in early universe cosmology. In addition, it may give experimental evidence for the use of Alcubierre's [7] (2008) expression of energy density as associated with 
gravitational waves.

\section{Nomenclature - Definitions}

$m_{n}($ Graviton $)=n / L+10^{-65}$ grams is a Kaluza Klein graviton mass expression, with a slight rest mass put in, for four dimensions, of $10^{-65}$ grams. The $\mathrm{n}$ is for nodes in 5 dimensions, and $\mathrm{L}$ is the length (size) of a 5 th dimension.

$n_{f}=$ numerical density of a group of gravitons, per unit phase space "volume" at a given frequency, $f$.

$\Omega_{g w} \equiv \rho_{g w} / \rho_{c}$ is a ratio of gravity wave "density" per unit volume of phase space, over a phase space volume.

$\Omega_{\text {effective }}$ here, in this case is the same as $\Omega_{g w}$ Pauli Fierz Langrangian $=$ classical stability and absence of ghosts lead directly to the standard Fierz-Pauli Lagrangian.

KK. = Kaluza-Klein. A model that seeks to unify the two fundamental forces of gravitation and electromagnetism In the case of this paper, it is for particles obeying a unification of gravitation and electromagnetism.

DM, DE = Dark Matter, and Dark energy. Non baryonic matter in cosmology.

$Z_{N}=$ partition function, a concept usually from statistical physics.

$\lambda=$ wavelength of a "particle". Frequently in association with matter as a particle and a wave, i.e. wave- particle duality of quantum mechanics.

\section{Acknowledgements}

The author wishes to thank Dr. Fangyu Li as well as Stuart Allen, of international media associates whom freed the author to think about physics, and get back to his work. This work is supported in part by National Nature Science Foundation of China grant No. 11075224.

\section{References}

[1] A. W. Beckwith, "Applications of Euclidian Snyder Geometry to the Foundations of Space-Time Physics," Electronic Journal of Theoretical Physics, Vol. 7, No. 24,
2010, pp. 241-266.

[2] A. Beckwith, "Energy Content of Gravitation as a Way to Quantify Both Entropy and Information Generation in the Early Universe," Journal of Modern Physics, Vol. 2, No. 2, 2011, pp. 58-61. doi:10.4236/jmp.2011.22010

[3] G. Smoot, "CMB Observations and the Standard Model of the Universe," International Programme of Cosmology Daniel Chalonge "Third Millennium," Paris, 2007.

[4] M. Maggiore, "Gravitational Waves, Volume 1: Theory and Experiment," Oxford University Press, Oxford, 2008.

[5] M. Visser, "Mass for the Graviton," General Relativity and Gravitation, Vol. 30, No. 12, 1998, pp. 1717-1728. doi:10.1023/A:1026611026766

[6] R. Durrer and M. Rinaldi, "Graviton Production in Noninflationary Cosmology," Physical Review D, Vol. 79, No. 6, 2009, p. 063507. doi:10.1103/PhysRevD.79.063507

[7] M. Alcubierre, "Introduction to Numerical Relativity," Oxford University Press, Oxford, 2008.

[8] Y. Ng, "Spacetime Foam: From Entropy and Holography to Infinite Statistics and Nonlocality," Entropy 2008, Vol. 10, No. 4, 2008, pp. 441-461. doi:10.3390/e10040441

[9] G. 't Hooft, "The Mathematical Basis for Deterministic Quantum Mechanics," In: Th. M. Nieuwenhuizen, et al., Eds., Beyond the Quantum, World Scientific Publishing, Singapore, 2006.

[10] G. 't Hooft, "Determinism beneath Quantum Mechanics," Report Number: ITP-02/69; SPIN-2002/45. http://arxiv.org/abs/quant-ph/0212095

[11] R. Maartens, "Braneworld Cosmology, Chapter 7 - The Physics of the Early Universe," Lecture Notes in Physics, Vol. 653, 2005, pp. 213-252.

[12] U. Sarkar, "Particle and Astroparticle Physics in "Series of High Energy Physics, Cosmology and Gravitation'," Taylor \& Francis, New York, 2008.

[13] T. Ortin, "Gravity and Strings," Cambridge University Press, Oxford, 2007.

[14] J. Y. Kim, "Stability and Fluctuation Modes of Giant Gravitons with NSNS B Field," Physics Letters B, Vol. 529, No. 1-2, 2002, pp. 150-162. doi:10.1016/S0370-2693(02)01233-9

[15] Lloyd, S., "Computational Capacity of the Universe," Physical Review Letters, Vol. 88, No. 23, 2002, p. 237901. doi:10.1103/PhysRevLett.88.237901 